\title{
Interleukin-1 receptor antagonist (anakinra) treatment in patients with systemic-onset juvenile idiopathic arthritis or adult onset Still disease: preliminary experience in France
}

\author{
T Lequerré, ${ }^{1}$ P Quartier, ${ }^{2}$ D Rosellini, ${ }^{2}$ F Alaoui, ${ }^{3}$ M De Bandt, ${ }^{4} 0$ Mejjad, ${ }^{1}$ I Kone-Paut, ${ }^{5}$ \\ M Michel, ${ }^{6}$ E Dernis, ${ }^{7}$ M Khellaf, ${ }^{6} \mathrm{~N}$ Limal, ${ }^{8} \mathrm{C}$ Job-Deslandre, ${ }^{9} \mathrm{~B}$ Fautrel, ${ }^{10} \mathrm{X}$ Le Loët, ${ }^{1}$ \\ $\mathrm{J}$ Sibilia, ${ }^{3}$ on behalf of the Société Francophone pour la Rhumatologie et les Maladies \\ Inflammatoires en Pédiatrie (SOFREMIP) and the Club Rhumatismes et Inflammation \\ (CRI)
}

\begin{abstract}
See editorial on p 281
${ }^{1}$ Rheumatology Department, Rouen University Hospital \& Inserm 905, Rouen, France;

${ }^{2}$ Paediatric Immuno-

Haematology and Rheumatology Department, Necker-Enfants

Malades Hospital, Paris, France;

${ }^{3}$ Rheumatology Department,

CHU Strasbourg, Strasbourg,

France; ${ }^{4}$ Rheumatology

Department, Hôpital Robert

Ballanger, Aulnay sous Bois,

France; ${ }^{5}$ Paediatric Department,

Bicêtre Hospital, Le Kremlin-

Bicêtre, France; ${ }^{6}$ Department of

Internal Medicine, Hôpital Henri

Mondor, Créteil, France;

${ }^{7}$ Rheumatology Department,

Hôpital Le Mans, Le Mans,

France; ${ }^{8}$ Department of Internal

Medicine, Hôpital Pitié

Salpétrière, France;

${ }^{9}$ Rheumatology A Department, Cochin Hospital, Paris, France:

${ }^{10}$ Rheumatology Department, Hôpital Pitié-Salpétrière, Paris, France
\end{abstract}

Correspondence to:

T. Lequerré, Rheumatology Department, Rouen University Hospital \& Inserm 905, 76031 Rouen, France; thierry.

lequerre@univ-rouen.fr. Pierre

Quartier, Unité d'ImmunologieHématologie et Rhumatologie pédiatrique, Hôpital NeckerEnfants Malades, 149 rue de Sèvres, 75015 Paris, France; quartier@necker.fr

$\mathrm{TL}$ and $\mathrm{PQ}$ contributed equally to this work.

Accepted 23 September 2007 Published Online First

18 October 2007

\begin{abstract}
Background: Anakinra treatment has been reported to be effective in some patients with systemic-onset juvenile idiopathic arthritis (SoJIA) or adult-onset Still disease (AoSD).
\end{abstract}

Objectives: To assess the efficacy and the safety of anakinra treatment in SoJIA and AoSD.

Methods: SoJIA and AoSD patients were treated with anakinra (1-2 mg/kg/day in children, $100 \mathrm{mg} /$ day in adults); we analysed its effect on fever, erythrocyte sedimentation rate (ESR) and C-reactive protein (CRP) levels, numbers of swollen and tender joints, the assessment of disease activity (by physician and parent/ patient) and pain (by parent/patient), and American College of Rheumatology (ACR) pediatric core set criteria for JIA activity.

Results: A total of 35 patients were included, 20 with SoJIA and 15 with AoSD. Their mean age (range) at the onset of treatment was $12.4(3-23)$ and 38.1 (22-62) years, respectively; disease duration was $7.0(1-16)$ and 7.8 (2-27) years, respectively. Active arthritis was present in all cases but one. Of the 20 SoJIA patients, 5 achieved ACR 50\% improvement in symptoms (ACR50) response criteria at 6 months. Steroid dose had been decreased by $15 \%$ to $78 \%$ in 10 cases. A total of 11 of the 15 AoSD patients achieved at least a $50 \%$ improvement for all disease markers (mean follow-up: 17.5 (11-27) months). Steroids had been stopped in two cases and the dose was decreased by $45 \%$ to $95 \%$ in 12 patients. Two patients stopped anakinra due to severe skin reaction, and two patients due to infection: one visceral leishmaniasis and one varicella.

Conclusion: Anakinra was effective in most AoSD patients, but less than half SoJIA patients achieved a marked and sustained improvement.

Systemic-onset juvenile idiopathic arthritis (SoJIA) and adult-onset Still disease (AoSD) are rare systemic inflammatory disorders of unknown aetiology. They share similar features including fever, evanescent rash, arthralgia and arthritis, myalgia, lymphadenopathy, serositis, hyperleukocytosis, and elevated levels of erythrocyte sedimentation and C-reactive protein (CRP). Typically, serum ferritin levels are elevated and the percentage of glycosylated ferritinaemia is low. Corticosteroid treatment has for many years been the sole effective treatment in most patients with SoJIA or AoSD with a severe, polycyclic or persistent course. Resistance to corticosteroid treatment is uncommon, but it has been observed in some patients. Dependency on high-dose corticosteroid treatment is common. Longlasting corticosteroid therapy induces many complications including cataracts, bone demineralisation and growth impairment during childhood. However, the possibilities for minimising corticoid use in these patients is limited, as non-steroidal anti-inflammatory drugs (NSAIDs), intravenous immunoglobulins, methotrexate and even anti-tumour necrosis factor (TNF) $\alpha$ therapy often lack efficacy. ${ }^{1-3}$ Other treatments, such as azathioprin or gold salts, are poorly effective in most cases and may favour macrophage activation syndrome in SoJIA. Thalidomide and antiinterleukin (IL)-6 receptor antibody treatment have shown efficacy in small series of SoJIA patients. ${ }^{45}$

The pro-inflammatory cytokines, IL-18 and IL-1, may play a key role in SoJIA and AoSD pathogenesis. $^{67}$ Marked efficacy of IL-1 receptor antagonist (IL-1Ra) (anakinra) treatment has been reported in some SoJIA or AoSD patients. ${ }^{6}{ }^{8}$ The largest study published thus far for SoJIA patients undergoing anakinra treatment included nine children with an active disease that did not respond to previous treatment. Eight of the nine patients responded well to anakinra treatment, and treatment resolved most clinical manifestations resulting in the normalisation of their biological markers for inflammation. ${ }^{6}$ Fitzgerald et al described six AoSD patients refractory to methotrexate and prednisone $(\mathrm{n}=4)$ or etanercept treatment $(\mathrm{n}=2)$. All patients responded quickly and positively, and demonstrated a regression of clinical symptoms and a normalisation of haematological and biochemical markers for systemic disease. ${ }^{9}$ Two French organisations, the "Société Francophone pour la Rhumatologie et les Maladies Inflammatoires en Pédiatrie" and the "Club Rhumatismes et Inflammation" (a section of the French Society of Rheumatology dedicated to the study of inflammatory rheumatisms), carried out a joint survey to assess the efficacy and tolerability of anakinra treatment in a larger cohort of patients with either SoJIA or AoSD. 


\section{PATIENTS AND METHODS}

\section{Patient selection and data collection}

Patients with SoJIA or AoSD treated with anakinra in France before 31 December 2005, and having at least one assessment of treatment efficacy and safety following treatment onset were eligible for this study. SoJIA patients had to fulfil the International League Against Rheumatism (ILAR) criteria for SoJIA. ${ }^{11}$ AoSD patients had to fulfil the Yamaguchi criteria for AoSD. ${ }^{12}$ Patients and paediatric patient's parents were informed that anakinra treatment had only been approved for adults patients with rheumatoid arthritis. Oral informed consent was obtained in all cases before starting treatment.

Paediatricians retrospectively collected data relating to their patients before starting treatment; data was subsequently collected on a monthly basis. Rheumatologists filled in a standardised questionnaire sent online with the support of the "Club Rhumatisme et Inflammation" (http://www.cri-net. com). In the standardised form, rheumatologists had to check precisely that patients had fulfilled the Yamaguchi criteria for AoSD (with standard definition for fever $=$ at least $39^{\circ} \mathrm{C}$ for 1 week or more; arthralgia $=$ at least 2 weeks duration). During the follow-up, there was a standard definition for fever (at least $39^{\circ} \mathrm{C}$ ), but not for other clinical data, given that only experienced rheumatologists took part in this study. Data were retrospectively analysed at 3 months, 6 months and at the latest follow-up. The data was collected or the notes were reviewed using a standard form, and they were then analysed by three of the authors (DR, TL, PQ).

Data collected for SoJIA and AoSD patients included sex, date of birth, disease duration, previous treatments, systemic symptoms (fever, exanthaema, lymphadenopathy, hepatosplenomegaly, and serositis), tender joint count (TJC), swollen joint count (SJC), physician global assessment of disease activity, patient assessment of pain for adults, and parents' assessment of pain and disease activity for children. The following laboratory values were collected: haematological profile, firsthour erythrocyte sedimentation rate (ESR), CRP, and serum ferritin levels. The percentage of glycosylated ferritin was also recorded, when available. The result of the Childhood Health Assessment Questionnaire (CHAO) for each SoJIA patient was recorded at each visit.

\section{Assessment of anakinra treatment efficacy and tolerability}

We analysed the effect of anakinra treatment on systemic features including fever, skin rash, ESR or CRP levels, and on other disease markers before starting treatment (TJC, SJC, physician's and patient's or parent's assessment of disease activity or pain on a visual analogue scale) at 3 months, 6 months and at the latest follow-up. Response was defined as a resolution of systemic symptoms and an improvement of the American College of Rheumatology (ACR) score by at least 20\% in patients with AoSD. Response in patients with SoJIA was defined as the resolution of systemic symptoms and the improvement of Giannini's ACR pediatric (pedi) core set criteria by at least $30 \%$ for polyarticular JIA activity assessment. ${ }^{13}$ Giannini's ACR pedi core set of response variables included: the number of active joints (joint swelling or, in the absence of swelling, limited range of motion accompanied by either pain or tenderness), number of joints with limited range of motion, physician global assessment of disease severity (by $100 \mathrm{~mm}$ visual analogue scale (VAS)), parent global assessment of patient overall well-being (100 mm VAS), functional ability with the validated translated version of the Childhood Health Assessment Questionnaire Disability Index (CHAQ-DI), and
ESR. ACR pedi 30 improvement was defined as $\geqslant 30 \%$ improvement in at least three of the six JIA core response variables and $\geqslant 30 \%$ worsening in not more than one of the variables. ACR pedi 50 and 70 improvements were defined as $\geqslant 50 \%$ and $70 \%$ improvement respectively in at least three of the six JIA core response variables and $\geqslant 30 \%$ worsening in not more than one of the variables. The response was defined as "partial response" if the ACR or ACR pedi score improvement was less than $50 \%$, and was defined as "complete response" if the ACR or ACR pedi score improved by $50 \%$ or more. The changes in serum ferritin levels and in the percentage of glycosylated ferritin were also analysed, when available. We also analysed the extent to which the dose of corticosteroids could be decreased. Adverse events for each patient were systematically recorded from the onset of anakinra treatment to the last visit.

\section{Statistical analysis}

Data are expressed as mean (SD). Intention-to-treat analyses were used: percentages of improvement of each clinical and biological marker were analysed at 3 months, 6 months and at the latest follow-up under anakinra treatment. Differences between marker values before and after treatment were analysed using either a two-sided $t$ test or the Wilcoxon matched-pairs test for nonparametric data at a significance level of 0.05

\section{RESULTS}

\section{Characteristics of SoJIA and AoSD patients at the initiation of} anakinra treatment

A total of 35 patients were included, 20 with SoJIA and 15 with AoSD. The main characteristics of the patients at the start of anakinra treatment are listed in tables 1 and 2 . All patients were treated with corticosteroids before starting anakinra treatment. The mean duration of corticosteroid treatment was 5.7 years for SoJIA and 4.6 years for AoSD patients. In total, 20 patients with SoJIA and 12 with AoSD were on steroid treatment at the start of anakinra treatment. Active disease persisted under steroid treatment, and corticodependance was present in all cases. The mean predniso(lo)ne dose was $0.50(0.32) \mathrm{mg} / \mathrm{kg}$ for SoJIA and 26.8 (20.1) mg for AoSD patients. Previous disease-modifying antirheumatic drugs (DMARDs) had been used in all patients, except the youngest child. They were noted as being ineffective, or not very effective. Previous DMARDs, including methotrexate (MTX), had been used in 34 cases (lack of efficacy in 29 patients, hepatic side-effects in 3 patients, digestive or cardiac side-effects in 5 patients), and anti-TNF $\alpha$ therapy had been used in 24 cases (lack of efficacy in 20 patients and infusion reactions in 4 patients). Thalidomide was at least partly effective in four children; however, abnormal electromyography findings led to the withdrawal of this treatment.

Fever was present in only 9 of the 20 patients with SoJIA and in 13 of 15 patients with AoSD at the start of anakinra treatment (table 2). However, three SoJIA patients with no fever had skin rash daily. Active arthritis was present in all cases but one child, who was undergoing treatment with high-dose steroids. The mean numbers of swollen and tender joints were 18.1 and 20.1, respectively, in patients with SoJIA, and 10.7 and 9.9 in AoSD patients. Radiological evidence confirmed irreversible joint damage in six patients with SoJIA and five with AoSD. Among SoJIA patients, 2 had active systemic features with no or few active joint involvement, 10 had chronic arthritis with systemic flares, and 8 no longer suffered systemic symptoms but had severe, diffuse joint involvement. Among AoSD patients, 10 had a chronic arthritic form with systemic 
Table 1 Patients' main characteristics at anakinra treatment initiation

\begin{tabular}{lll}
\hline Characteristics & SoJIA (n= 20) & AoSD (n= 15) \\
\hline Gender (M/F) & $8 / 12$ & $4 / 11$ \\
Age, years (mean (SD)) & $12.4(5.2)$ & $38.1(12.8)$ \\
Disease duration, years (mean (SD)) & $7.0(4)$ & $7.8(6.4)$ \\
Systemic features (no. of patients) & $12(60 \%)$ & $13(87 \%)$ \\
Fever & $9(45 \%)$ & $13(87 \%)$ \\
Evanescent rash* & $8(40 \%)$ & $8(53 \%)$ \\
Serositis & $3(15 \%)$ & $2(13 \%)$ \\
Previous treatments with DMARDs (no. of & & \\
patients): & & \\
MTX广 & $19(95 \%)$ & $15(100 \%)$ \\
Anti-TNF $\alpha$ & $14(70 \%)$ & $10(67 \%)$ \\
Thalidomide & $5(25 \%)$ & $2(13 \%)$ \\
IVIG & $0(0 \%)$ & $5(33 \%)$ \\
Cyclosporin & $6(30 \%)$ & $0(0 \%)$ \\
Other DMARDs: & $2(10 \%)$ & $6(40 \%)$ \\
Ongoing predniso(lo)ne daily dose & $0.50(0.32) \mathrm{mg} / \mathrm{kg}$ & $26.8(20.1) \mathrm{mg}$ \\
\hline
\end{tabular}

*In three patients with SoJIA, evanescent rash was present in the absence of fever. $\dagger$ The average and maximum doses of MTX received prior to anakinra treatment were $0.6 \mathrm{mg} / \mathrm{kg} / \mathrm{week}$ and $1 \mathrm{mg} / \mathrm{kg} /$ week, respectively. The average and maximum doses of MTX received prior to anakinra treatment were $20 \mathrm{mg} /$ week and $30 \mathrm{mg} /$ week respectively.

\$Rituximab $(n=1)$ and cyclophosphamide $(n=1)$ for SoJIA patients; rituximab $(n=2)$, cyclophosphamide $(n=1)$, mycophenolate mofetil $(n=1)$, azathioprin $(n=1)$ and sulphasalazin $(n=1)$ for AoSD pateints.

AoSD, adult onset Still disease; DMARDs : disease-modifying antirheumatic drugs; IVIG, intravenous immunoglobulins; MTX, methotrexate; SoJIA, systemic onset juvenile idiopathic arthritis; TNF, tumour necrosis factor.

flares, 3 had a systemic form, and 2 patients showed no systemic symptoms but an active articular involvement.

Five SoJIA and 11 AoSD patients were administered anakinra in combination with MTX $(n=14)$ or another DMARD $(n=2)$ (table 2).

\section{Efficacy of anakinra in SoJIA and AoSD patients}

The mean follow-up (range) from the initiation of treatment to the latest follow-up was 14.7 months (2-27) in SoJIA patients and 14.3 months (1-27) in AoSD patients.

Anakinra was started at doses from $1-2 \mathrm{mg} / \mathrm{kg} / \mathrm{day}$ (maximum $100 \mathrm{mg}$ /day) in SoJIA patients and it was administered at a dose of $100 \mathrm{mg} /$ day in AoSD patients. After the second month, only one of five SoJIA patients, who were receiving a dose of $1 \mathrm{mg} / \mathrm{kg}$, showed significant improvement; a higher dose, $2 \mathrm{mg} / \mathrm{kg}$ (maximum of a $100 \mathrm{mg}$ ) once a day, was administered to the other four patients. The dose was subsequently increased to $100 \mathrm{mg}$ twice a day for one of the adult SoJIA patients (see table 2, patient 2).

Of the 20 SoJIA patients, 15 initially showed at least some improvement under anakinra treatment. Clinical systemic features, including fever and rash, were resolved in 14 cases within the first 3 months. However, the percentage of patients who achieved a 30, 50 and $70 \%$ improvement, according to ACR pedi core set criteria, were $55 \%, 30 \%$ and $0 \%$ at 3 months, respectively; $50 \%, 25 \%$ and $10 \%$ at 6 months, respectively; and $45 \%, 20 \%$ and $10 \%$ at the latest follow-up, respectively (12 to 27 months from the start of anakinra treatment). The steroid dose had been reduced by $15 \%$ to $78 \%$ at 6 months in relation to the baseline in nine patients (see below). Serum ferritin levels and percentages of glycosylated ferritin in six SoJIA patients were documented on day 1 and during the follow-up. At the start of treatment, all cases had high levels of serum ferritin, five patients had low percentages of glycosylated ferritin (0 to $20 \%$, $\mathrm{n}>25$ ), and one patient with active systemic features displayed normal percentages. Serum ferritin values in patients undergoing anakinra treatment decreased in all cases. The percentage of glycosylated ferritin increased in the five patients who initially had low values and normalised in four of them between months 3 and 6 of treatment.

A total of 11 of the 15 AoSD patients (73\%) had a prompt and dramatic improvement in all disease markers (fig 1) despite the severity of their disease, and were still on anakinra treatment at the latest visit, in December 2006 (follow-up in these patients was between 11 and 27 months). All clinical and biological markers in 9 of the $15(60 \%)$ patients improved by at least $50 \%$ at 6 months (fig 1). A total of 11 patients responded to anakinra: 9 of the 11 patients achieved a complete response at 3 months; 10 of the 11 patients, at 6 months; and 9 of the 11 patients, at the latest follow-up. Two patients had a partial response (displaying fever or elevated ESR or CRP) at the latest follow-up. Corticosteroids had been stopped in 2 of the 11 patients that responded to treatment and the dose could be reduced by $45 \%$ to $95 \%$ in relation to the baseline in 8 patients (fig 2).

Anakinra was associated with an improvement of most disease variables at the latest follow-up (16 months on average) (table 3). No obvious discrepancies were observed between changes in several disease markers in SoJIA or in AoSD patients. The response to anakinra treatment in SoJIA patients did not seem to correlate to any pre-treatment variable; for example the age at which anakinra treatment was started, the disease duration, or the presence or absence of active systemic features at enrolment.

Some patients initially received methotrexate or other DMARDS in combination with anakinra: 2 of the 5 SoJIA patients and 7 of the 12 AoSD patients continued methotrexate; 1 SoJIA and 1 AoSD patient started another DMARD. Of the 12 AoSD patients, 9 achieved CR and 1 patient achieved PR at 6 months; 8 of these patients were still complete responders at the latest follow-up and 2 were partial responders. However, only one of the five SoJIA patients treated with a combination of anakinra and another DMARD achieved sustained improvement.

\section{Adverse events and treatment withdrawal}

Two SoJIA patients stopped anakinra treatment during the first 3 months, and three patients stopped treatment between the months 3 and 6 . Treatment withdrawal, in these five patients, was either due to intolerance (one case) or a lack of efficacy (four cases). Four AoSD patients stopped anakinra because of side-effects $(n=2)$ or a lack of efficacy $(n=2)$.

A single serious adverse event occurred in a child who developed a visceral Leishmania infection during the month 6 of anakinra treatment. ${ }^{14}$ The child was living in an endemic zone in the south of France, but her pre-therapeutic blood sample was negative. Anakinra treatment was stopped, and treatment specific for Leishmania was started and resulted in a favourable outcome. SoJIA became active again following anakinra treatment withdrawal; therefore, anakinra was recently restarted in this patient. We recorded various other infectious events in SoJIA patients: one case of varicella, two cases of rhinoparyngitis and one case of non-extensive labial herpes. We recorded the following for AoSD patients: one bronchitis case, one uncomplicated hepatitis A case, one varicella and one cutaneous infection case (after a piercing, leading to transient anakinra withdrawal). One patient had osteonecrosis of the femoral hip, possibly due to long-lasting corticosteroid treatment. 
Table 2 Patients' characteristics and treatment at the onset and at the latest follow-up of anakinra treatment

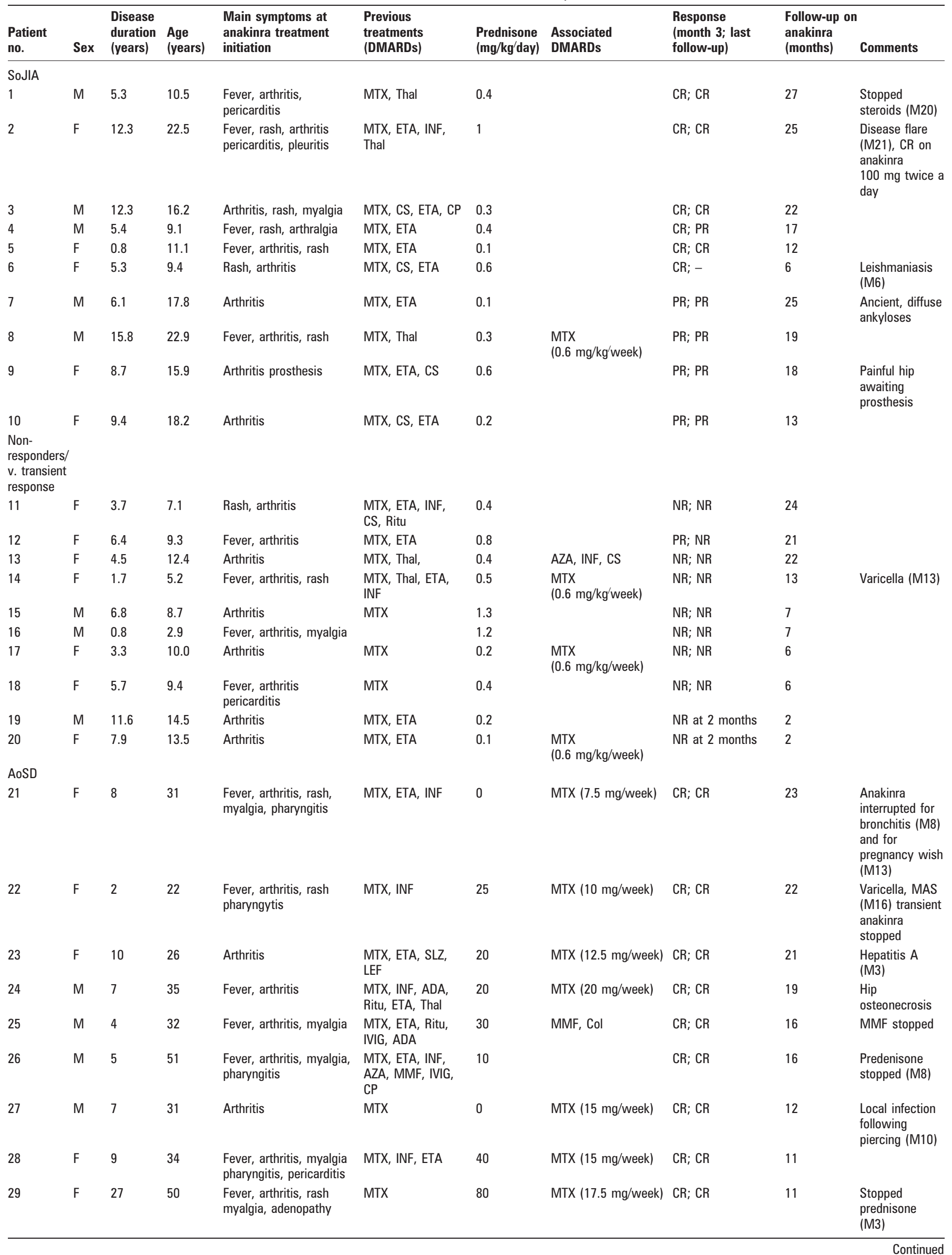


Table 2 Continued

\begin{tabular}{|c|c|c|c|c|c|c|c|c|c|c|}
\hline $\begin{array}{l}\text { Patient } \\
\text { no. }\end{array}$ & Sex & $\begin{array}{l}\text { Disease } \\
\text { duration } \\
\text { (years) }\end{array}$ & $\begin{array}{l}\text { Age } \\
\text { (years) }\end{array}$ & $\begin{array}{l}\text { Main symptoms at } \\
\text { anakinra treatment } \\
\text { initiation }\end{array}$ & $\begin{array}{l}\text { Previous } \\
\text { treatments } \\
\text { (DMARDs) }\end{array}$ & $\begin{array}{l}\text { Prednisone } \\
\text { (mg//kg'day) }\end{array}$ & $\begin{array}{l}\text { Associated } \\
\text { DMARDs }\end{array}$ & $\begin{array}{l}\text { Response } \\
\text { (month 3; last } \\
\text { follow-up) }\end{array}$ & $\begin{array}{l}\text { Follow-up on } \\
\text { anakinra } \\
\text { (months) }\end{array}$ & Comments \\
\hline 30 & $\mathrm{~F}$ & 4 & 62 & $\begin{array}{l}\text { Fever, arthritis, rash, } \\
\text { myalgia }\end{array}$ & MTX, SLZ, INF & 40 & & PR; PR & 27 & \\
\hline 31 & $\mathrm{~F}$ & 3 & 47 & Fever, arthritis, rash & MTX & 30 & Col & PR; PR & 15 & \\
\hline \multicolumn{11}{|l|}{$\begin{array}{l}\text { Non- } \\
\text { responders }\end{array}$} \\
\hline 32 & $\mathrm{~F}$ & 5 & 62 & Fever, arthritis, pleuresis & MTX, INF, IVIG & 40 & MTX (20 mg/week) & $N R ; N R$ & 9 & $\begin{array}{l}\text { Anakinra } \\
\text { stopped for lack } \\
\text { of efficacy (M8) }\end{array}$ \\
\hline 33 & $\mathrm{~F}$ & 4 & 24 & $\begin{array}{l}\text { Fever, arthritis, rash, } \\
\text { myalgia, pharyngytis, } \\
\text { adenopathy }\end{array}$ & MTX, IVIG & 20 & MTX (20 mg/week) & $N R ; N R$ & 8 & $\begin{array}{l}\text { Only transient } \\
\text { efficacy }\end{array}$ \\
\hline 34 & $\mathrm{~F}$ & 18 & 40 & $\begin{array}{l}\text { Fever, arthritis, myalgia, } \\
\text { rash }\end{array}$ & MTX, Thal, ETA & 0 & & Intolerance & 1 & $\begin{array}{l}\text { Severe skin } \\
\text { rash (day 10) }\end{array}$ \\
\hline 35 & $\mathrm{~F}$ & 4 & 25 & $\begin{array}{l}\text { Fever, arthritis, rash } \\
\text { pharyngitis }\end{array}$ & MTX, IVIG & 20 & MTX (20 mg/week) & Intolerance & $<1$ & $\begin{array}{l}\text { Anakinra } \\
\text { stopped (M1) } \\
\text { (injection site } \\
\text { reactions) }\end{array}$ \\
\hline
\end{tabular}

ADA, adalimumab; AoSD, adult-onset Still disease; AZA, azathioprine; Col, colchicine; $C P$, cyclophosphamide; $C R$, complete response: no systemic symptoms and at least $50 \%$ improvement of ACR score (AoSD) or ACR pediatric score (SoJIA); CS, cyclosporin; DMARDs, disease-modifying antirheumatic drugs; ETA, etanercept; INF, infliximab; IVIG, intravenous immunoglobulins; M(n), month no.; MAS, macrophage activation syndrome; MMF, mycophenolate mofetil; MTX, methotrexate; NR, non-responder; PR, partial response: no systemic symptoms and 20 to $49 \%$ improvement of ACR score (AOSD) or 30 to $49 \%$ improvement of ACR pediatric score (SoJIA); Ritu, rituximab; SLZ, sulphasalazin; SoJIA, Systemic-onset juvenile idiopathic arthritis; Thal, thalidomide.

Local pain or reactions to injections were the most frequent adverse events. All but two cases reported pain during injections in SoJIA patients; pain was also recorded in one AoSD patient. SoJIA patients also experienced local inflammation at the injection site or pruritus during the first few weeks, with a favourable outcome afterwards. Two AoSD patients developed a skin rash after 1 month and 3 months, respectively, leading to the withdrawal of anakinra. Two SoJIA patients experienced transient headaches.

\section{DISCUSSION}

We assessed the efficacy and tolerability of administering treatment with anakinra in SoJIA and AoSD patients. This is the largest such series and the first to analyse the effects of this treatment on SoJIA and AoSD patients, in parallel. We recorded similar results for SoJIA and AoSD patients in terms of disease duration and the main markers for disease activity. However, fever was more consistently present in AoSD patients at the start of anakinra treatment.

\section{- At least $70 \%$ improvement from baseline $\quad \square 50-69 \% \quad \square 20-49 \%$}
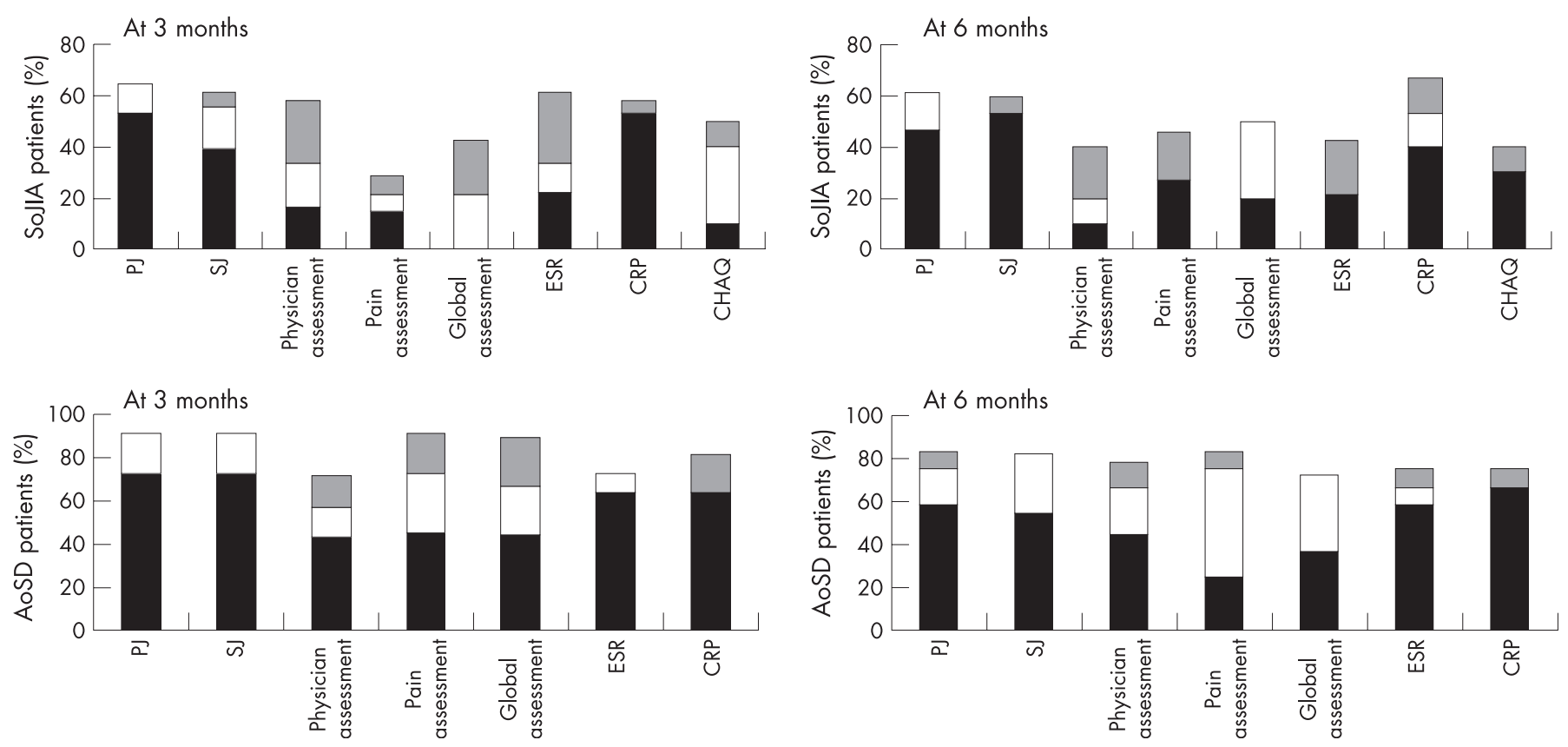

Figure 1 SoJIA and AoSD patients' response to anakinra in an intention-to-treat analysis at 3 and 6 months. Percentage of SoJIA patients (top) and AoSD patients (bottom) who achieved a 20\%,50\% and 70\% improvement in the main disease activity variables at 3 and 6 months in an intention-totreat analysis. AoSD, adult-onset Still disease; CHAQ, Childhood Health Assessment Questionnaire; CRP, C-reactive protein; ESR, erythrocyte sedimentation rate; SJ, swollen joint count; SoJIA, systemic-onset juvenile idiopathic arthritis; TJ, tender joint count. Pain and disease activity levels for children were assessed by their parents. 

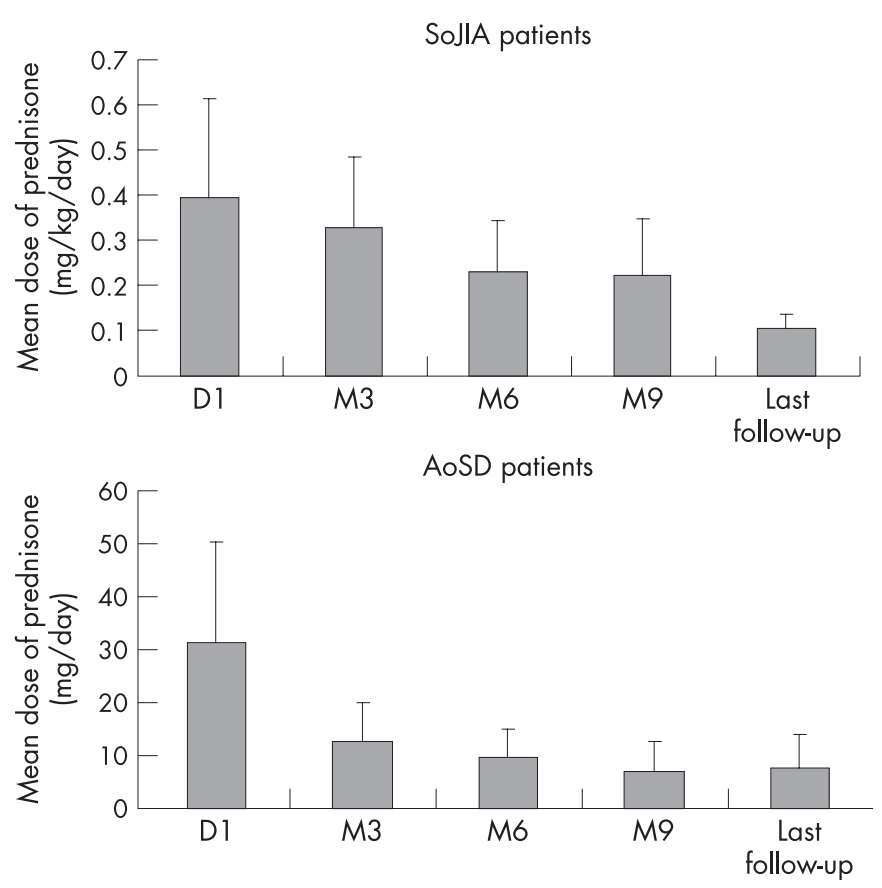

Figure 2 Changes over time of the daily prednisone dose in patients that responded to anakinra. Mean (SD) of the daily prednisone dose in responders to anakinra before treatment, at 3, 6, 9 months, and at the latest follow-up. Top: SoJIA patients. Bottom: AoSD patients. AoSD, adult-onset Still disease; SoJIA, systemic-onset juvenile idiopathic arthritis.

Response to anakinra treatment was rapid and sustained in most patients with AoSD and in a significant proportion of SoJIA patients, confirming previously published data and providing evidence suggesting that IL-1 is a key cytokine in these two diseases. ${ }^{6-10}$ In at least five patients with SoJIA and four with AoSD, fever and pain was resolved within 3 and 10 days, respectively, and in some cases was resolved within $24 \mathrm{~h}$. Here, AoSD patients responded well to anakinra treatment; however, these patients were pre-treated with large doses of various drugs (three DMARDs on average prior to anakinra). Moreover, most AoSD patients (8/10) who did not respond to anti-TNF $\alpha$ treatment responded to anakinra. By contrast, the proportion of SoJIA patients who responded to anakinra in our series, and the magnitude of their response were not so high. The proportion of SoJIA patients that responded to treatment was also lower than in the study of Pascual et al, in which eight of nine patients presented a dramatic improvement with anakinra. ${ }^{6}$ In this study, 8 of 12 SoJIA patients, who had fever and/or a skin rash at the start of anakinra treatment, showed at least some improvement in systemic features and fulfilled ACR pedi 30 response criteria to anakinra treatment; however, less than half achieved a 50\% improvement using the ACR pedi core set criteria, and the proportion of SoJIA patients achieving ACR pedi 30 and ACR pedi 50 improvements were only $45 \%$ and $20 \%$ at the latest follow-up, respectively.

Several differences between AoSD and SoJIA patients in our series may account for the higher rate of response achieved by adults. First, fever and systemic symptoms were more consistently present in adults. Neutralisation of IL-1 might be more effective in patients with highly active systemic disease than in patients with chronic arthritis, with no or less intense systemic symptoms. However, the presence of fever and rash in SoJIA patients at enrolment was not associated with a higher response rate in our series. Second, the number of swollen and tender joints was higher on average in SoJIA patients, than in AoSD patients. Conversely, there is no clear evidence that using anakinra in combination with another DMARD, such as methotrexate, improves its efficacy in SoJIA patients: only one of five SoJIA patients treated with a combination of anakinra and methotrexate or azathioprine achieved partial response. Another issue to address is whether the anakinra dose or the number of injections per day should have been increased in patients that did not respond. Little is known about the pharmacokinetics of anakinra in children. In other autoinflammatory diseases, such as neonatal onset multisystem inflammatory disease (NOMID)/chronic infantile neurological, cutaneous, and articular (CINCA) syndrome and hyper-IgD syndromes, some patients require injections twice a day; this was also the case in one SoJIA patient in our study.

Table 3 Clinical and biological variables before and at the latest follow-up for patients undergoing anakinra treatment

\begin{tabular}{|c|c|c|c|c|c|c|}
\hline & \multicolumn{3}{|l|}{ SoJIA $(n=20)$} & \multicolumn{3}{|l|}{ AoSD $(n=15)$} \\
\hline & At anakinra onset & $\begin{array}{l}\text { Last visit* on } \\
\text { anakinra }\end{array}$ & p Value & At anakinra onset & $\begin{array}{l}\text { Last visit* on } \\
\text { anakinra }\end{array}$ & p Value \\
\hline Tender joint count & $20.5(14.7)$ & $9.9(22.5)$ & 0.02 & $8.5(5.9)$ & $1.5(2.7)$ & 0.0002 \\
\hline Swollen joint count & $18.1(15.1)$ & $10.7(19.7)$ & 0.01 & $5.9(5.8)$ & $0.9(1.5)$ & 0.0005 \\
\hline Assessment of pain $(0-10 \mathrm{VAS}) \uparrow$ & $4.4(3.0)$ & $4.1(34.3)$ & $0.3 \%$ & $6.6(2.2)$ & $1.8(2.5)$ & $0.0002 \%$ \\
\hline $\begin{array}{l}\text { Global assessment of disease activity } \\
(0-10 \text { VAS }) \dagger\end{array}$ & 4.1 (3.3) & $3.5(31.9)$ & $0.16 t$ & 7 (1.9) & $1.7(2.3)$ & 0.0005 \\
\hline $\begin{array}{l}\text { Physician global assessment of disease } \\
\text { activity (0-10 VAS) }\end{array}$ & $4.3(2.6)$ & $3.8(30.6)$ & $0.02 \div$ & $6.9(2)$ & $2(2.3)$ & $0.002 \div$ \\
\hline $\mathrm{ESR}, \mathrm{mm} / \mathrm{h}$ & $51.9(28.8)$ & $24.4(20.2)$ & $<0.0001$ & $74(33.5)$ & $22.1(24.6)$ & 0.0005 \\
\hline CRP, mg/litre & $78.9(42.3)$ & $25.5(29.9)$ & 0.0006 & $91.9(71.8)$ & $16.6(20.6)$ & 0.001 \\
\hline Ferritinaemia, ng/ml & $2672(5640)$ & ND & & $997(1410)$ & $283(419)$ & 0.094 \\
\hline Leukocyte countsł (109/litre) & $15.4(4.7)$ & $10.7(4.2)$ & $0.004 \%$ & $11.9(6.1)$ & $7.5(2.3)$ & $0.017 末$ \\
\hline CHAO & $1.4(1.0)$ & $0.6(1.0)$ & 0.01 & ND & ND & ND \\
\hline Corticosteroid doses & $0.50(0.32) \S$ & $0.24(0.22) \S$ & 0.05 & $26.8(20.1) \S$ & $8.6(7.6)$ & 0.0047 \\
\hline
\end{tabular}

Values indicated are mean (SD). Except where indicated otherwise, means of each variable before and after anakinra were compared by a non-parametric Wilcoxon test. *Estimated by parents for SoJIA patients and by patient for AoSD patients.

\$Means were compared by $t$ test. $p$ Values $<0.05$ were considered significant.

thatest follow-up: 14.7 (2-27) months in SoJIA patients and 14.3 (2-27) in AoSD patients

$\S \mathrm{ln} \mathrm{mg} / \mathrm{kg} / \mathrm{day}$ in SoJIA patients and in $\mathrm{mg} /$ day in AoSD patients.

AoSD, adult-onset Still disease; CHAO, Childhood Health Assessment Questionnaire; CRP, C-reactive protein; ESR, erythrocyte sedimentation rate; SoJIA, systemic-onset juvenile idiopathic arthritis; VAS, visual analogue scale. 
One of the main goals of anakinra treatment, particularly in growing children, is to decrease the dose of corticosteroids. Here, corticodependency was markedly reduced in 12 of 15 AoSD patients, but only in 9 of 20 SoJIA patients. This is consistent with a lower proportion of SoJIA patients responding to treatment. Nevertheless, in SoJIA patients whose daily predniso(lo)ne dose was tapered, a marked dose reduction was achieved. Pascual et al reported in SoJIA patients treated with anakinra that oral prednisone was stopped in one patient and tapered in the other patients; this again was consistent with the robust response demonstrated by those patients responding to anakinra treatment.

This study corroborates the sustained efficacy and safety of anakinra treatment in, at least, a proportion of AoSD and SoJIA patients resistant to conventional drugs, including methotrexate and TNF- $\alpha$ blocking agents. Lower levels of corticosteroid were required for patients responding to anakinra treatment. The lower response rate observed in SoJIA patients indicates that prospective, randomised, and controlled trials are needed, assessing, in particular, the pharmacokinetics of anakinra in children.

Acknowledgements: The authors acknowledge the assistance of Drs Anne-Marie Prieur, Xavier Mariette, Christelle Sordet, Anne Pagnier, Richard Mouy and Nathalie Costedoat-Chalumeau.

Competing interests: None declared.

\section{REFERENCES}

1. Quartier $\mathbf{P}$, Taupin $\mathrm{P}$, Bourdeaut $\mathrm{F}$, Lemelle I, Pillet $\mathrm{P}$, Bost $\mathrm{M}$, et al. Efficacy of etanercept for the treatment of juvenile idiopathic arthritis according to the onset type. Arthritis Rheum 2003;48:1093-101.
2. Fautrel B, Sibilia J, Mariette X, Combe B. Club Rhumatismes et Inflammation. Tumour necrosis factor alpha blocking agents in refractory adult Still's disease: an observational study of 20 cases. Ann Rheum Dis 2005;64:262-6.

3. Horneff G, Schmeling H, Biedermann T, Foeldvari I, Ganser G, Girschick HJ, et al. The German etanercept registry for treatment of juvenile idiopathic arthritis. Ann Rheum Dis 2004;63:1638-44.

4. Lehman TJ, Schechter SJ, Sundel RP, Oliveira SK, Huttenlocher A, Onel KB. Thalidomide for severe systemic onset juvenile rheumatoid arthritis: A multicenter study. J Pediatr 2004;145:856-7.

5. Yokota S, Miyamae T, Imagawa T, Iwata N, Katakura S, Mori M, et al. Therapeutic efficacy of humanized recombinant anti-interleukin-6 receptor antibody in children with systemic-onset juvenile idiopathic arthritis. Arthritis Rheum 2005;52:818-25

6. Pascual V, Allantaz F, Arce E, Punaro M, Banchereau J. Role of interleukin-1 (IL-1) in the pathogenesis of systemic onset juvenile idiopathic arthritis and clinical response to IL-1 blockade. J Exp Med 2005:201:1479-86.

7. Fujii T, Nojima T, Yasuoka H, Satoh S, Nakamura K, Kuwana M, et al. Cytokine and immunogenetic profiles in Japanese patients with adult-onset Still's disease. Association with chronic articular disease. Rheumatology 2001;40:1398-404.

8. Verbsky JW, White AJ. Effective use of the recombinant interleukin 1 receptor antagonist anakinra in therapy resistant systemic onset juvenile rheumatoid arthritis. J Rheumatol 2004;31:2071-5.

9. Fitzgerald AA, Leclercq SA, Yan A, Homik JE, Dinarello CA. Rapid responses to anakinra in patients with refractory adult-onset Still's disease. Arthritis Rheum 2005;52:1794-803.

10. Vasques Godinho FM, Parreira Santos MJ, Canas da Silva J. Refractory adult onset Still's disease successfully treated with anakinra. Ann Rheum Dis 2005:64:647-8.

11. Petty RE, Southwood TR, Manners P, Baum J, Glass DN, Goldenberg J, et al. International League of Associations for Rheumatology. International League of Associations for Rheumatology classification of juvenile idiopathic arthritis: second revision, Edmonton, 2001. J Rheumatol 2004;31:390-2.

12. Yamaguchi M, Ohta A, Tsunematsu T, Kasukawa R, Mizushima $Y$, Kashiwagi $H$, et al. Preliminary criteria for classification of adult Still's disease. J Rheumatol 1992;19:424-30.

13. Giannini EH, Ruperto N, Ravelli A, Lovell DJ, Felson DT, Martini A. Preliminary definition of improvement in juvenile arthritis. Arthritis Rheum 1997;40:1202-9.

14. Kone-Paut I, Retornaz K, Garnier JM, Bader-Meunier B. Visceral Leishmaniasis in a patient with systemic juvenile arthritis treated by IL-1Ra agonist (anakinra). Clin Exp Rheumatol 2007;25:119.

bmjupdates+

bmjupdates+ is a unique and free alerting service, designed to keep you up to date with the medical literature that is truly important to your practice.

bmjupdates+ will alert you to important new research and will provide you with the best new evidence concerning important advances in health care, tailored to your medical interests and time demands.

Where does the information come from?

bmjupdates+ applies an expert critical appraisal filter to over 100 top medical journals A panel of over 2000 physicians find the few 'must read' studies for each area of clinical interest

Sign up to receive your tailored email alerts, searching access and more...

www.bmjupdates.com 